\title{
ANALYSIS OF THICK BEAM USING NEW HYPERBOLIC SHEAR DEFORMATION THEORY
}

\author{
Mithun. K. Sawant ${ }^{1}$, Ajay. G. Dahake ${ }^{2}$, Rohit. S. Deshmukh ${ }^{3}$, Vikas. N. Nimbalkar ${ }^{4}$ \\ ${ }^{1}$ Assistant Professor, Civil Engineering Department, Dr. D. Y. Patil Institute of engineering, Management \& \\ Research, Akurdi-Pune, Maharashtra, India \\ ${ }^{2}$ Associate Professor, Civil Engineering Department, Marathwada Institute of Technology, Aurangabad, \\ Maharashtra, India \\ ${ }^{3}$ Assistant Professor, Civil Engineering Department, Dr. D. Y. Patil Institute of engineering, Management \& \\ Research, Akurdi-Pune, Maharashtra, India \\ ${ }^{4}$ Assistant Professor, Civil Engineering Department, Dr. D. Y. Patil Institute of engineering, Management \& \\ Research, Akurdi-Pune, Maharashtra, India
}

\begin{abstract}
A new hyperbolic shear deformation theory for flexure of thick beams, in which number of variables is same as that in the hyperbolic shear deformation theory, is developed. The theory takes into account transverse shear deformation effects; the noteworthy feature of theory is that the transverse shear stresses can be obtained directly from the use of constitutive relations with efficacy, satisfying the shear stress free condition on the top and bottom surfaces of the beam. Hence, the theory obviates the need of shear correction factor. The cantilever isotropic beam subjected to varying load is examined using the present theory. Results obtained are discussed critically with those of other theories.
\end{abstract}

Keywords: Thick Beam, Hyperbolic Shear Deformation, Principle of Virtual Work, Equilibrium Equations, Displacement, Stress

\section{INTRODUCTION}

It is well-known that elementary theory of bending of beam based on Euler-Bernoulli hypothesis disregards the effects of the shear deformation and stress concentration. The theory is suitable for slender beams and is not suitable for thick or deep beams since it is based on the assumption that the sections normal to neutral axis before bending remain so during bending and after bending, implying that the transverse shear strain is zero. Since theory neglects the transverse shear deformation, it underestimates deflections in case of thick beams where shear deformation effects are significant.

Rayleigh [9] and Timoshenko [10] were the pioneer investigators to include refined effects such as rotatory inertia and shear deformation in the beam theory. Timoshenko showed that the effect of transverse shear is much greater than that of rotatory inertia on the response of transverse vibration of prismatic bars. This theory is now widely referred to as Timoshenko beam theory or first order shear deformation theory (FSDT) in the literature. The first order shear deformation theory (FSDT) of Timoshenko [11] includes refined effects .such as the rotatory inertia and shear deformation in the beam theory. Timoshenko showed that the effect of transverse shear is much greater than that of rotatory inertia on the response of transverse vibration of prismatic bars. In this theory transverse shear strain distribution is assumed to be constant through the beam thickness and thus requires shear correction factor to appropriately represent the strain energy of deformation. Cowper [3] has given refined expression for the shear correction factor for different cross-sections of the beam.

The discrepancies in the elementary theory of beam bending and first order shear deformation theory forced the development of higher order or equivalent refined shear deformation theories. Levinson [8], Krishna Murty [7], Baluch, et.al [1], Bhimaraddi and Chandrashekhara [2] presented parabolic shear deformation theories assuming a higher variation of axial displacement in terms of thickness coordinate. These theories satisfy shear stress free boundary conditions on the top and bottom surfaces of the beam and thus obviate the need of shear correction factor. Heyliger and Reddy [6] presented higher order shear deformation theories for the static and free vibration The theories based on trigonometric and hyperbolic functions to represent the shear de-formation effects through the thickness is the another class of refined theories. However, with these theories shear stress free boundary conditions are not satisfied at top and bottom surfaces of the beam. This discrepancy is removed by Ghugal and Shimpi [4] and developed a variationally consistent refined trigonometric shear deformation theory for flexure and free vibration of thick isotropic beams. Ghugal and Sharma [5] developed the variationally consistent hyperbolic shear deformation theory for flexure analysis of thick beams and obtained the displacements, stresses and fundamental frequencies of flexure mode and thickness shear modes from free vibration 
of simply supported beams. In this paper, a variationally consistent hyperbolic shear deformation theory previously developed by Ghugal and Sharma [5] for thick beams is used to obtain the general bending solutions for thick isotropic beams. The theory is applied to uniform isotropic solid beams of rectangular cross-section for static flexure with various boundary and loading conditions. The results are compared with those of elementary, refined beam theory to verify the credibility of the present shear deformation theory.

In this paper development of theory and its application to thick cantilever beam is presented.

\section{DEVELOPMENT OF THEORY}

The beam under consideration as shown in Figure1 occupies in $0-x-y-z$ Cartesian coordinate system the region:

$$
\mathrm{O} \leq x \leq L ; \quad 0 \leq y \leq b ; \quad-\frac{h}{2} \leq z \leq \frac{h}{2}
$$

where $\mathrm{x}, \mathrm{y}, \mathrm{z}$ are Cartesian coordinates, $\mathrm{L}$ and $\mathrm{b}$ are the length and width of beam in the $\mathrm{x}$ and $\mathrm{y}$ directions respectively, and $h$ is the thickness of the beam in the $\mathrm{z}$ direction. The beam is made up of homogeneous, linearly elastic isotropic material.

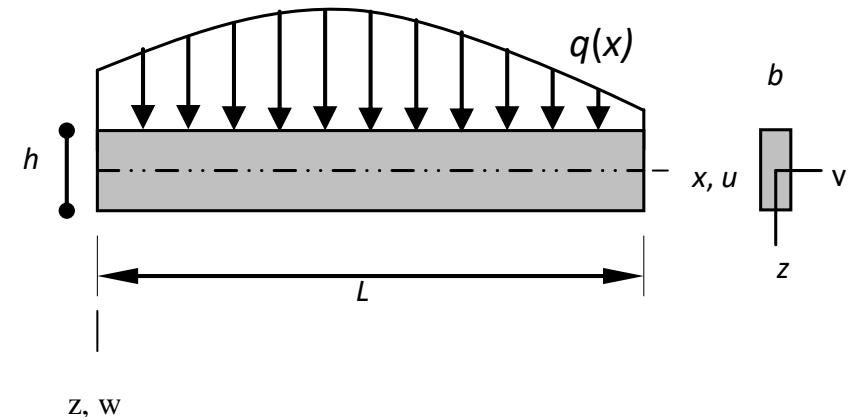

Fig. 1: Beam under bending in $\mathrm{x}-\mathrm{z}$ plane

\subsection{The Displacement Field}

The displacement field of the present beam theory is of the form:

$$
u(x, z)=-z \frac{d w}{d x}+\left[\begin{array}{c}
z \cosh \left(\frac{\alpha}{2}\right)- \\
\frac{h}{\alpha} \sinh \left(\frac{\alpha z}{h}\right)
\end{array}\right]
$$

$$
w(x, z)=w(x)
$$

where $u$ the axial displacement in $\mathrm{x}$ direction and $\mathrm{w}$ is the transverse displacement in $\mathrm{z}$ direction of the beam. The sinusoidal function is assigned according to the shear stress distribution through the thickness of the beam. The function $\phi$ represents rotation of the beam at neutral axis, which is an unknown function to be determined. The normal and shear strains obtained within the framework of linear theory of elasticity using displacement field given by Eqn. (1) are as follows.

Shear strain: $\gamma_{z x}=\frac{\partial u}{\partial z}+\frac{d w}{d x}=\cos \frac{\pi z}{h} \phi$

The stress-strain relationships used are as follows: $\tau_{z x}=G \gamma_{z x}$

\subsection{Governing Equations and Boundary Conditions}

Using the expressions for strains and stresses (2) through (4) and using the principle of virtual work, variationally consistent governing differential equations and boundary conditions for the beam under consideration can be obtained. The principle of virtual work when applied to the beam leads to:

$$
\begin{aligned}
& b \int_{x=0}^{x=L} \int_{z=-h / 2}^{z=+h / 2}\left(\sigma_{x} \delta \varepsilon_{x}+\tau_{z x} \delta \gamma_{z x}\right) d x d z \\
& -\int_{x=0}^{x=L} q(x) \delta w d x=0
\end{aligned}
$$

where the symbol $\delta$ denotes the variational operator. Employing Green's theorem in Eqn. (4) successively, we obtain the coupled Euler-Lagrange equations which are the governing differential equations and associated boundary conditions of the beam. The governing differential equations obtained are as follows:

$$
\begin{aligned}
& E I \frac{d^{4} w}{d x^{4}}-\frac{24}{\pi^{3}} E I \frac{d^{3} \phi}{d x^{3}}=q(x) \\
& \frac{24}{\pi^{3}} E I \frac{d^{3} w}{d x^{3}}-\frac{6}{\pi^{2}} E I \frac{d^{2} \phi}{d x^{2}}+\frac{G A}{2} \phi=0
\end{aligned}
$$

The associated consistent natural boundary condition obtained is of following form:

At the ends $\mathrm{x}=0$ and $\mathrm{x}=\mathrm{L}$

$V_{x}=E I \frac{d^{3} w}{d x^{3}}-\frac{24}{\pi^{3}} E I \frac{d^{2} \phi}{d x^{2}}=0$ or $w$ is prescribed

$M_{x}=E I \frac{d^{2} w}{d x^{2}}-\frac{24}{\pi^{3}} E I \frac{d \phi}{d x}=0$ or $\frac{d w}{d x}$ is prescribed

$M_{a}=E I \frac{24}{\pi^{3}} \frac{d^{2} w}{d x^{2}}-\frac{6}{\pi^{2}} E I \frac{d \phi}{d x}=0$ or $\phi$ is prescribed

\subsection{The General Solution of Governing}

\section{Equilibrium Equations of the Beam}

The general solution for transverse displacement $\mathrm{w}(\mathrm{x})$ and warping function $\phi(x)$ is obtained using Eqns. (6) and (7) using method of solution of linear differential equations with constant coefficients. Integrating and rearranging the first governing Eqn. (6), we obtain the following equation

$\frac{d^{3} w}{d x^{3}}=\frac{24}{\pi^{3}} \frac{d^{2} \phi}{d x^{2}}+\frac{Q(x)}{E I}$

where $\mathrm{Q}(\mathrm{x})$ is the generalized shear force for beam and it is

given by $Q(x)=\int_{0}^{x} q d x+C_{1}$. 
Now the second governing Eqn. (7) is rearranged in the following form:

$$
\frac{d^{3} w}{d x^{3}}=\frac{\pi}{4} \frac{d^{2} \phi}{d x^{2}}-\beta \phi
$$

A single equation in terms of $\phi$ is now obtained using Eqns. (11) and (12) as:

$$
\frac{d^{2} \phi}{d x^{2}}-\lambda^{2} \phi=\frac{Q(x)}{\alpha E I}
$$

where constants $\alpha, \beta$ and $\lambda$ in Eqns. (11) and (12) are as follows

$$
\alpha=\left(\frac{\pi}{4}-\frac{24}{\pi^{3}}\right), \beta=\left(\frac{\pi^{3}}{48} \frac{G A}{E I}\right) \text { and } \lambda^{2}=\frac{\beta}{\alpha}
$$

The general solution of Eqn. (13) is as follows:

$$
\phi(x)=C_{2} \cosh \lambda x+C_{3} \sinh \lambda x-\frac{Q(x)}{\beta E I}
$$

The equation of transverse displacement $w(x)$ is obtained by substituting the expression of $\phi(x)$ in Eqn. (12) and then integrating it thrice with respect to $x$. The general solution for $w(x)$ is obtained as follows:

$$
\begin{aligned}
& E I w(x)=\iiint \int q d x d x d x d x+\frac{C_{1} x^{3}}{6}+ \\
& \left(\frac{\pi}{4} \lambda^{2}-\beta\right) \frac{E I}{\lambda^{3}}\left(C_{2} \sinh \lambda x+C_{3} \cosh \lambda x\right)+C_{4} \frac{x^{2}}{2}+C_{5} x+C_{6}
\end{aligned}
$$

where $C_{1}, C_{2}, C_{3}, C_{4}, C_{5}$ and $C_{6}$ are arbitrary constants and can be obtained by imposing boundary conditions of beam.

\section{ILLUSTRATIVE EXAMPLES}

In order to prove the efficacy of the present theory, the following numerical examples are considered. The material properties for beam used are: $\mathrm{E}=210 \mathrm{GPa}, \mu=0.3$ and $\rho=$ $7800 \mathrm{~kg} / \mathrm{m} 3$, where $\mathrm{E}$ is the Young's modulus, $\rho$ is the density, and $\mu$ is the Poisson's ratio of beam material.

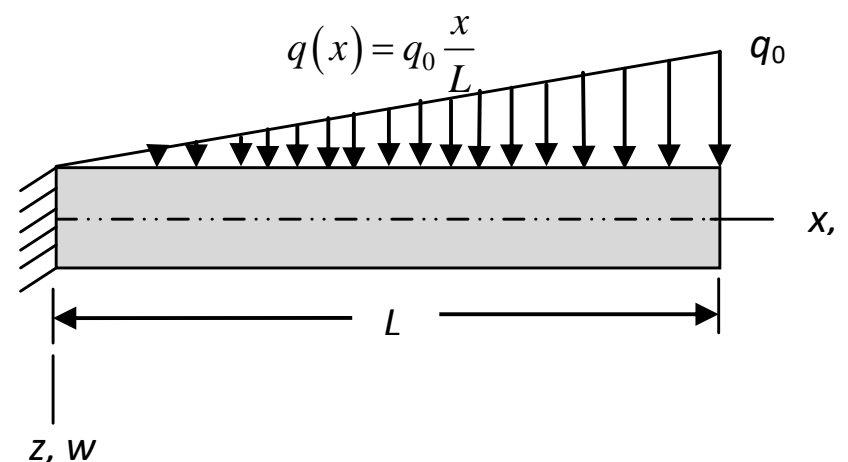

Fig. 3.2: Cantilever beam with varying load

General expressions obtained are as follows:

$$
\begin{aligned}
& w(x)=\frac{q L^{4}}{120 E I}\left\{\begin{array}{l}
\frac{x^{5}}{L^{5}}-10 \frac{x^{3}}{L^{3}}+20 \frac{x^{2}}{L^{2}}+\frac{B_{0}}{C_{0}} \frac{E}{G} \frac{h^{2}}{L^{2}}\left(\frac{5}{3} \frac{x^{3}}{l^{3}}-5 \frac{x^{2}}{l^{2}}\right)- \\
\frac{A_{0}^{2}}{C_{0}} \frac{E}{G} \frac{h^{2}}{L^{2}} 5\left[\frac{(\zeta(x)+1)}{\lambda L}-\frac{x}{L}\right]
\end{array}\right\} \\
& u=\frac{q h}{E b}\left\{\begin{array}{l}
-\frac{1}{10} \frac{z}{h} \frac{L^{3}}{h^{3}}\left[\begin{array}{l}
5 \frac{x^{4}}{L^{4}}-30 \frac{x^{2}}{L^{2}}-40 \frac{x}{L}-\frac{B_{0}}{C_{0}} \frac{E}{G} \frac{h^{2}}{L^{2}} \\
\left(5 \frac{x^{2}}{L^{2}}-10 \frac{x}{L}\right)-\frac{A_{0}^{2}}{C_{0}} \frac{E}{G} \frac{h^{2}}{L^{2}} 5(-\zeta(x)-1)
\end{array}\right]+ \\
{\left[\frac{z}{h} \operatorname{ch}\left(\frac{\alpha}{2}\right)-\frac{1}{\alpha} \operatorname{sh}\left(\frac{\alpha z}{h}\right)\right] \frac{A_{0}}{C_{0}} \frac{E}{G} \frac{1}{2} \frac{l}{h}\left(\begin{array}{l}
1-\frac{x^{2}}{L^{2}} \\
+\zeta(x)
\end{array}\right)}
\end{array}\right\} \\
& \sigma_{x}=\frac{q}{b}\left\{\begin{array}{l}
-\frac{z}{h} \frac{1}{10} \frac{L^{2}}{h^{2}}\left[\begin{array}{l}
20 \frac{x^{3}}{L^{3}}-60 \frac{x}{L}+40-\frac{B_{0}}{C_{0}} \frac{E}{G} \frac{h^{2}}{l^{2}} \\
\left(10 \frac{x}{L}-10\right)-\frac{A_{0}^{2}}{C_{0}} \frac{E}{G} \frac{h^{2}}{l^{2}} 5 \lambda L \zeta(x)
\end{array}\right]+ \\
{\left[\frac{z}{h} \operatorname{ch}\left(\frac{\alpha}{2}\right)-\frac{1}{\alpha} \operatorname{sh}\left(\frac{\alpha z}{h}\right)\right] \frac{A_{0}}{C_{0}} \frac{E}{G} \frac{1}{2}\left[\lambda L(-\zeta(x))-2 \frac{x}{L}\right]}
\end{array}\right\} \\
& \tau_{z x}=\frac{A_{0}}{C_{0}} \frac{1}{2} \frac{q}{b} \frac{L}{h}\left[\operatorname{ch}\left(\frac{\alpha}{2}\right)-\operatorname{ch}\left(\frac{\alpha z}{h}\right)\right]\left(1+\zeta(x)-\frac{x^{2}}{L^{2}}\right)
\end{aligned}
$$

\subsection{RESULTS AND DISCUSSION}

The results for maximum transverse displacement and maximum transverse shear stresses are presented in the following non dimensional form for the purpose of presenting the results in this paper,

$$
\bar{u}=\frac{E b u}{q h}, \quad \bar{w}=\frac{10 E b h^{3} w}{q L^{4}}, \quad \bar{\sigma}_{x}=\frac{b \sigma_{x}}{q}, \quad \bar{\tau}_{z x}=\frac{b \tau_{z x}}{q}
$$

Table-1:Non-Dimensional Axial Displacement $(\bar{u})$ At $(X$ $=L, Z=H / 2)$, Transverse Deflection $(\bar{w})$ At $(X=L$, $Z=0.0)$ Axial Stress $\left(\bar{\sigma}_{x}\right)$ At $(X=0, Z=H / 2)$ and Maximum Transverse Shears Stresses $\bar{\tau}_{z x}^{C R}(X=0.01 l, Z$ $=0.0$ )

The results obtained by present new hyperbolic shear deformation theory are compared with those of elementary theory of beam bending (ETB), FSDT of Timoshenko, and HPSDT of Ghugal and Sharma. It is to be noted that the exact results from theory of elasticity are not available for the problems analyzed in this paper.

The comparison of results of maximum non-dimensional transverse displacement and shear stresses for the aspect ratios of 4 and 10 is presented in Table-I for beam subjected to varying load. Among the results of all the other theories, the values of present theory are in excellent agreement with the values of other refined theories for aspect ratio 4 and 10 except those of classical beam theory (ETB) and FSDT of Timoshenko. 


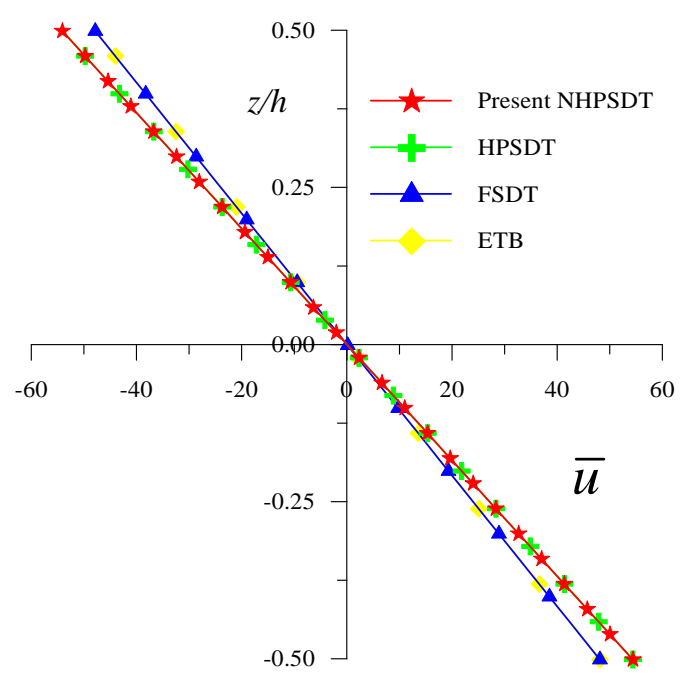

Graph 1: Variation of maximum axial displacement $(u)$ at $(S=4$ and $S=10)$

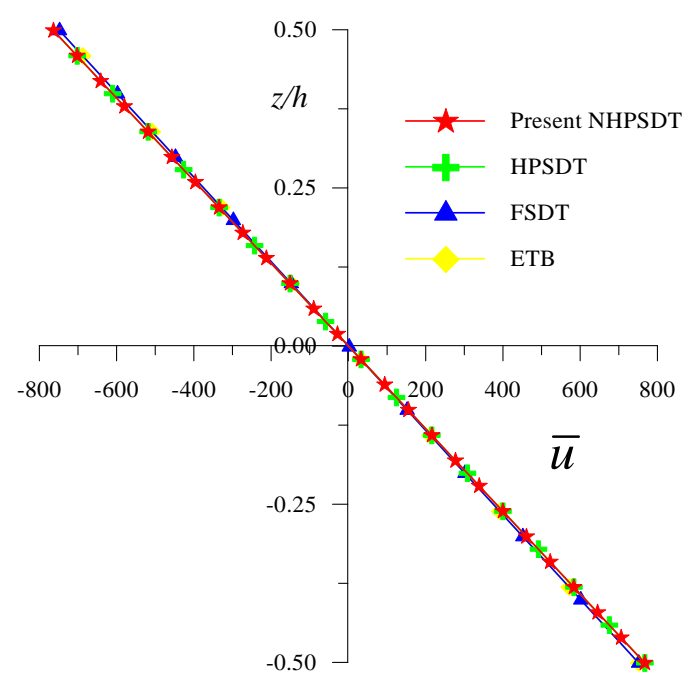

Graph 2: Variation of maximum transverse displacement (w) of cantilever beam at $(x=L, z=0)$ when subjected to varying load with aspect ratio AR.

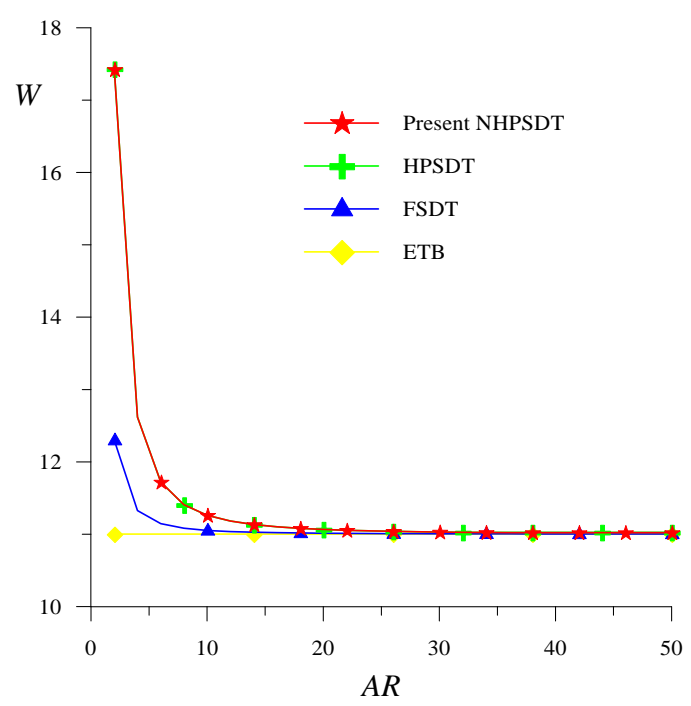

Graph 3: Variation of maximum axial stress $\left(\sigma_{x}\right)(S=4$ and $S=10)$
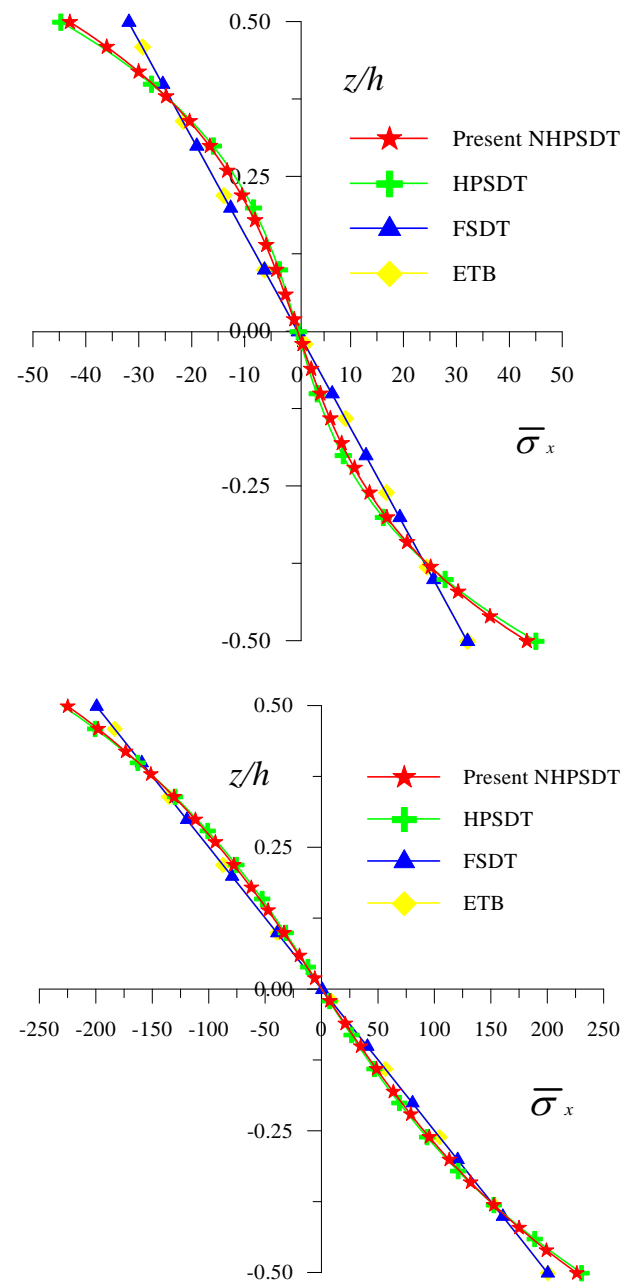

Graph 4: Variation of maximum transverse shear stress $\left(\tau_{z x}\right)$ $(S=4$ and $S=10)$
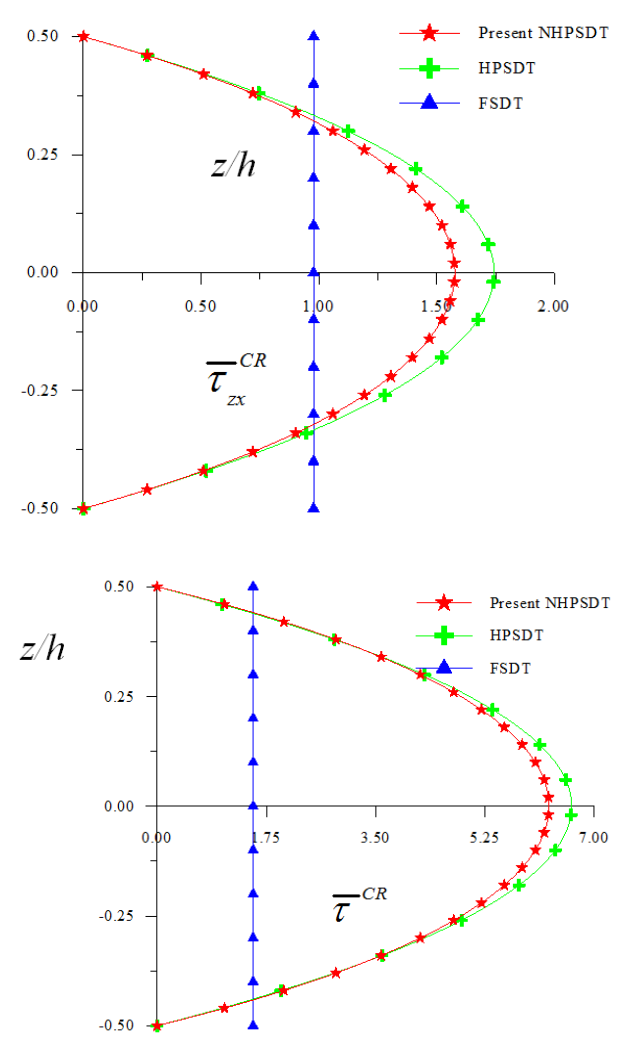


\begin{tabular}{|l|c|c|c|c|c|c|c|c|c|}
\hline \multirow{2}{*}{ Source } & \multirow{2}{*}{ Model } & \multicolumn{2}{|c|}{$\bar{w}$} & \multicolumn{2}{|c|}{$\bar{u}$} & \multicolumn{2}{c|}{$\bar{\sigma}_{x}$} & \multicolumn{2}{c|}{$\bar{\tau}_{\text {zx }}$} \\
\cline { 3 - 10 } & & $S=4$ & $S=10$ & $S=4$ & $S=10$ & $S=4$ & $S=10$ & $S=4$ & $S=10$ \\
\hline Present & NHPSDT & 17.422 & 11.260 & 54.2693 & 765.6731 & 43.1743 & 225.5727 & 1.5774 & 6.2768 \\
\hline Ghugal and Sharma [4] & HPSDT & 17.430 & 11.260 & 54.2637 & 765.6593 & 44.8807 & 229.8435 & 1.7440 & 6.6375 \\
\hline Timoshenko [11] & FSDT & 12.300 & 11.052 & 48.0000 & 750.0000 & 32.0000 & 200.0000 & 0.9772 & 1.5373 \\
\hline Bernoulli-Euler & ETB & 11.000 & 11.000 & 48.0000 & 750.0000 & 32.0000 & 200.0000 & - & - \\
\hline
\end{tabular}

\section{CONCLUSIONS}

The variationally consistent theoretical formulation of the theory with general solution technique of governing differential equations is presented. The general solutions for beam with varying load are obtained in case of thick cantilever beam. The displacements and shear stresses obtained by present theory are in excellent agreement with those of other equivalent refined and higher order theories. The present theory yields the realistic variation of transverse displacement through and shear stresses the thickness of beam.

\section{ACKNOWLEDGEMENT}

I am greatly indebted forever to my guide Dr. A.G. Dahake, Asso. Prof. Marathwada Institute of Technology, Aurangabad for his continuous encouragement, support, ideas, most constructive suggestions, valuable advice and confidence in me. I sincerely thank to Dr. Y.M. Ghugal, Prof. and Head of Applied Mechanics Department, Government College of Engineering, Karad for their encouragement and kind support and stimulating advice.

\section{REFERENCES}

[1] Baluch, M. H., Azad, A. K. and Khidir, M. A. Technical theory of beams with normal strain, ASCE J. of Engineering Mechanics, 0110(8), p.1233-37, 1984.

[2] Bhimaraddi, A., and Chandrashekhara, K. Observations on higher order beam Theory, ASCE J. of Aerospace Engineering, 6(4), p.408-413, 1993.

[3] Cowper, G. R. On the accuracy of Timoshenko beam theory, ASCE J. Engineering Mechanics Division. 94 (EM6), p.1447-53, 1968.

[4] Ghugal, Y. M. and Shmipi, R. P. A review of refined shear deformation theories for isotropic and anisotropic laminated beams, J. Reinforced Plastics And Composites, 20(3), p. 255-72, 2001.

[5] Ghugal, Y. M. and Sharma, R. A hyperbolic shear deformation theory for flexure and vibration of thick isotropic beams, International J. of Computational Methods, 6(4), p.585-604, 2009.

[6] Heyliger, P. R. and Reddy, J. N. A higher order beam finite element for bending and vibration problems, J. Sound and Vibration, 126(2), p.309-326, 1988.

[7] Krishna Murthy, A. V. Towards a consistent beam theory, AIAA Journal, 22(6), p.811-16, 1984.

[8] Levinson, M. A. new rectangular beam theory, J. Sound and Vibration, 74(1), p.81-87, 1981.
[9] Lord Rayleigh J. W. S. The Theory of Sound, Macmillan Publishers, London, 1877.

[10] Timoshenko, S. P. On the correction for shear of the differential equation for transverse vibrations of prismatic bars, Philosophical Magazine, 41 (6), p. 74246, 1921.

[11] Timoshenko, S. P. and Goodier, J. N. Theory of Elasticity, McGraw-Hill, $3^{\text {rd }}$ Edition, Singapore. 1970.

\section{BIOGRAPHIES}

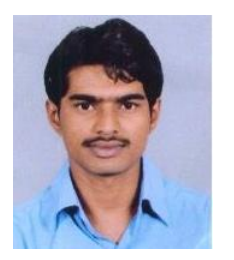

Mr. M.K. Sawant has obtained his BE (Civil) and ME (Struct.) from from Dr. B.A.M.U. Aurangabad university. His areas of interest are Analysis of Structures, Design of Structures.

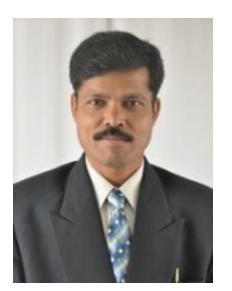

Dr. A.G. Dahake is presently working as Asso. Prof. in Civil Engineering Department, Marathwada Institute of Technology, Aurangabad. He has completed his ME (Struct.) and Ph.D. from Dr. B.A.M.U. Aurangabad. He has 11 years teaching experience and 4 years Civil works experience in Govt. His areas of interest are Concrete Technology, Solid mechanics, Structural Design and Analysis of Structures. He has published around 22 research papers at international/national level.

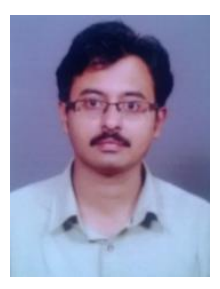

Mr. R.S. Deshmukh is presently working as Assistant Professor in Civil Engineering Department in Dr. D. Y. Patil Institute of engineering, Management and Research, Akurdi (Pune). He has pursued his ME (Construction Management) from SPPU. His areas of interest are Concrete Technology, Construction Management and Analysis of Structures.

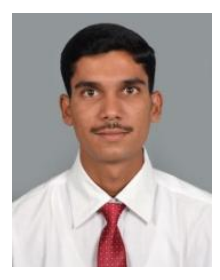

Mr. V.N. Nimbalkar has obtained his BE (Civil) and ME (Struct.) from from Dr. B.A.M.U. Aurangabad university. His areas of interest are Analysis of Structures, Design of Structures. 